\title{
Prognostic Value of c-MYC Expression in Patients with Peripheral Neuroblastic Tumors
}

\author{
Feng Zhang ${ }^{1-3}$ \\ Zhuo Tian ${ }^{4}$ \\ Tinghong $\mathrm{Li}^{5-7}$ \\ Xianjun $\mathrm{Hu}^{4}$ \\ Jin $\mathrm{Zhu}^{5-7}$
}

'Department of Otolaryngology, Children's Hospital of Chongqing Medical University, Chongqing, 400014, People's Republic of China; ${ }^{2}$ National Clinical Research Center for Child Health and Disorders, Chongqing, 4000I4, People's Republic of China; ${ }^{3}$ Ministry of Education Key Laboratory of Child Development and Disorders, Chongqing, 4000I4, People's Republic of China; ${ }^{4}$ Department of Geratology, Chongqing General Hospital, Chongqing, 4000I4, People's Republic of China; ${ }^{5}$ Department of Pathology, Chongqing Medical University, Chongqing, 400016, People's Republic of China; ${ }^{6}$ Institute of Neuroscience, Chongqing Medical University,

Chongqing, 400016, People's Republic of China; ${ }^{7}$ Key Laboratory of Neurobiology, Chongqing Medical University, Chongqing, 400016, People's Republic of China
Correspondence: Jin Zhu

Department of Pathology, Chongqing

Medical University, Chongqing, 4000I6,

People's Republic of China

Tel +86-23-68485789

Fax +86-23-63622874

Email fzhangltt5@I63.com
Objective: Neuroblastic tumors are the most common solid tumors in children. The aim of this study was to explore the prognostic value of immunostaining for cellularmyelocytomatosis viral oncogene (c-MYC) expression in patients with peripheral neuroblastic tumors (NTs).

Methods: A retrospective study was conducted to compare the expression of c-MYC detected by immunohistochemistry and v-myc avian myelocytomatosis viral oncogene neuroblastoma derived homolog $(M Y C N)$ by fluorescence in situ hybridization among 177 cases of NTs and determine the associations of c-MYC and $M Y C N$ with the clinical stages, morphological types, and survival rates of NTs.

Results: The cases positive for c-MYC were mainly the favorable histology type in stage 3 or 4 with a poor NT prognosis, but no morphological changes related to the poor prognosis were observed in their samples under a microscope. The cases with positive c-MYC expression did not overlap those with $M Y C N$ amplification.

Conclusion: Positive c-MYC expression portends a poor prognosis in patients with NTs. Keywords: peripheral neuroblastic tumors, c-MYC, immunostaining, $M Y C N$, prognosis

\section{Introduction}

Peripheral neuroblastic tumors (NTs), ie, solid tumors commonly found in children, can be classified into neuroblastoma, ganglioneuroblastoma, and ganglioneuroma tumors according to whether the proportion of nerve fibers is more than $50 \%$, whether there are neuroblasts or ganglion cells. ${ }^{1}$ Among these, neuroblastoma has the highest incidence rate, neuroblastoma was the most common infant malignancy $(6.5 / 100,000)$, followed by leukemia $(3.8 / 100,000)$, and brain and central nervous system tumors $(3.3 / 100,000)$, and has a severe health impact on patients, resulting in $15-20 \%$ of the deaths among the children who die of malignant tumors per year in the USA. $^{2}$ The prognosis of NTs is correlated with multiple factors. Shimada et al. ${ }^{3,4}$ have classified NTs into the favorable histology (FH) type and unfavorable histology (uFH) type based on the age-linked morphological changes. However, in the clinic, NTs are divided into different clinical stages based on the position, infiltration, and metastasis of tumors and then into different risk grades considering the results of clinical, pathological, and molecular tests. Recent in-depth research on the molecular characteristics of NTs has provided us with a much better understanding of the clinical features of the tumors, ${ }^{5}$ eg, that amplification of v-myc avian myelocytomatosis viral oncogene neuroblastoma derived homolog $(M Y C N)$ genes can decrease the survival rate and increase the rate of relapse and metastasis in patients with NTs. ${ }^{6,7}$ 
Even with the continuous improvement of treatment therapies, bad therapeutic efficacy is still common in some low-risk patients with pathological $\mathrm{FH}$ tumors in an early clinical stage, ${ }^{8}$ suggesting that the factors related to NT prognosis have not been fully explored. Recent studies have shown that the cellularmyelocytomatosis viral oncogene (c-MYC) protein can be expressed in neuroblastoma and is correlated with the morphological tumor type. ${ }^{9,10}$ In the present study, immunostaining for the c-MYC protein is performed after combining the histological subtypes of the patients and morphological characteristics of $M Y C N$ to explore the relationship between c-MYC expression and NT prognosis. The expression of c-myc indicates a poor prognosis. This study shows that c-myc expression in cases with relatively mild morphology is an important indicator of poor prognosis of neuroblastoma patients independent of age, MYCN, Shimada typing and other factors.

\section{Materials and Methods}

\section{Patient Cohort}

This retrospective study randomly enrolled 177 cases of NTs diagnosed within 2009-2012 at Chongqing Children's Hospital, Chongqing, China, and samples were obtained from those cases via biopsy or surgery before chemotherapy. These include 24 cases of ganglioneuroblastoma and 153 cases of neuroblastoma. In total, 108 boys and 69 girls were recruited, including $54<1.5$ years old, 89 between 1.5 and 5 years old, and $34>5$ years old. Among the participants, 56 had $\mathrm{uFH}$ and $121 \mathrm{FH}$ according to the International Neuroblastoma Pathology Classification (the Shimada classification). This study was carried out within an appropriate ethical framework. The patients' parents understood the scientific research uses, and all experimental procedures were approved by the Biomedical Ethics Committee of Chongqing Medical University.

\section{Hematoxylin and Eosin (HE) Staining}

Here, HE staining was conducted to assess the morphological changes. The tumor tissue was fixed with $4 \%$ paraformaldehyde before being dehydrated, dipped in wax, embedded, sectioned, and stained with HE. The morphological changes were assessed by pathologists from the Department of Pathology of Chongqing Medical University.

\section{Immunohistochemistry (IHC) and} Fluorescence in situ Hybridization (FISH) The c-MYC protein expression was detected immunohistochemically using formalin-fixed, paraffin-embedded sections with Leica BOND-MAX (Leica Biosystems, Mount Waverley, Australia). The sections were incubated with anti-c-MYC rabbit monoclonal antibody, clone Y69 (No. RMA-0664; Maixin Biotech. Ltd., Fuzhou, China), and counterstained with hematoxylin. This antibody is a working fluid and does not need to be diluted. A citrate buffer of $10 \mathrm{mM} \mathrm{pH6.0} \mathrm{was} \mathrm{used} \mathrm{for} \mathrm{high}$ pressure heating antigen repair. The incubation time was $30^{\circ} \mathrm{C}$ for 60 minutes. The secondary antibody KIT was purchased from Fuzhou Maixin Biotechnology Development Co., Ltd. (Elivision Super Kit, Kit-9922). Positive c-MYC expression was defined as diffuse brown/yellow staining in the nucleus of the cells, marked with $(+)$; weak positive c-MYC expression was defined as focal or light yellow staining in the nucleus of the cells, marked with $( \pm)$. Negative c-MYC expression was defined as no staining, marked with (-).

The MYCN gene amplification was detected with $M Y C N$ gene amplification kits (No. RM2235; Guangzhou Anbiping Medicine Technology Co., Ltd., Leica Microtome) and a ThermoBrite hybridizer (Leica Microsystems Inc., Germany). We counted over 100 tumor cells in a zone showing clear tumor tissue. If $M Y C N$ (red signal) clustering or red double minutes occurred, this suggested amplification of the $M Y C N$ genes. A ratio of $M Y C N$ (red signal) to $L A F$ (green signal) of 1.5 indicated a suspicious amplification that should be confirmed after considering the clinical results. A ratio of $M Y C N$ (red signal) to $L A F$ (green signal) of $\leq 1.0$ suggested a negative result.

\section{Morphological Type Related to Prognosis}

Based on the Shimada classification, the NTs were classified into four basic morphological categories: neuroblastoma, ganglioneuroblastoma intermixed, ganglioneuroblastoma nodular, and ganglioneuroma. Undifferentiated, poorly differentiated, and differentiated subtypes were included in the neuroblastoma category. Maturing and mature subtypes were included in the ganglioneuroma category. From the perspective of prognosis, the patients were divided into the $\mathrm{FH}$ and uFH groups after the patient age at first onset and the morphological subtype of the tumor tissue were considered.

\section{Statistical Analysis}

The data were analyzed using the SPSS software (version 17.0.1). Statistical differences were determined using an $\chi^{2}$ 
test or Fisher's test, and the significance level was $\alpha=0.05$. The Kappa test was used to check the consistency of the two statistical methods. The Kaplan-Meier curves, Log Rank tests, and Cox proportional hazards model survival analysis are used for survival analyses. Multivariate Cox Proportional Hazards Model Survival Analysis was used to comprehensively analyze whether c-myc expression was independent of other prognostic factors such as $\mathrm{MyCN}$, age and Shimada typing, and independently affected the prognosis.

\section{Results}

\section{MYCN Gene Expression Detected by FISH and c-MYC Protein Expression by IHC in NTs}

Figure 1 and Table 1 show the results of the MYCN expression identified by FISH and the c-MYC expression identified by IHC. Of the 177 enrolled cases, 28 had $M Y C N$ gene amplification, 16 had positive $(+)$ c-MYC
Table I MYCN Gene Expression Detected by FISH and c-MYC Protein Expression by IHC in NTs

\begin{tabular}{|l|l|l|l|l|}
\hline \multirow{2}{*}{ c-MYC } & \multicolumn{2}{|l|}{ MYCN } & \multirow{2}{*}{ Total } \\
\cline { 2 - 4 } & MNA & SAC & Non-MNA & \\
\hline$(+)$ & 0 & 1 & 15 & 16 \\
$( \pm)$ & 0 & 0 & 0 & 0 \\
$(-)$ & 28 & 4 & 129 & 161 \\
Total & 28 & 5 & 144 & 177 \\
\hline
\end{tabular}

Abbreviations: MNA, MYCN amplification; SAC, suspicious amplification cases; non-MNA, cases without MYCN amplification.

protein expression, 5 were cases suspected of MYCN amplification (including 1 case that showed positive c-MYC protein expression), and 129 had neither $M Y C N$ amplification nor positive c-MYC expression. Furthermore, amplified $M Y C N$ and positive c-MYC were never observed in the same case. After analysis by the Kappa test, the two statistical methods showed poor consistency, and the results were not correlated [Kappa $=-$
A
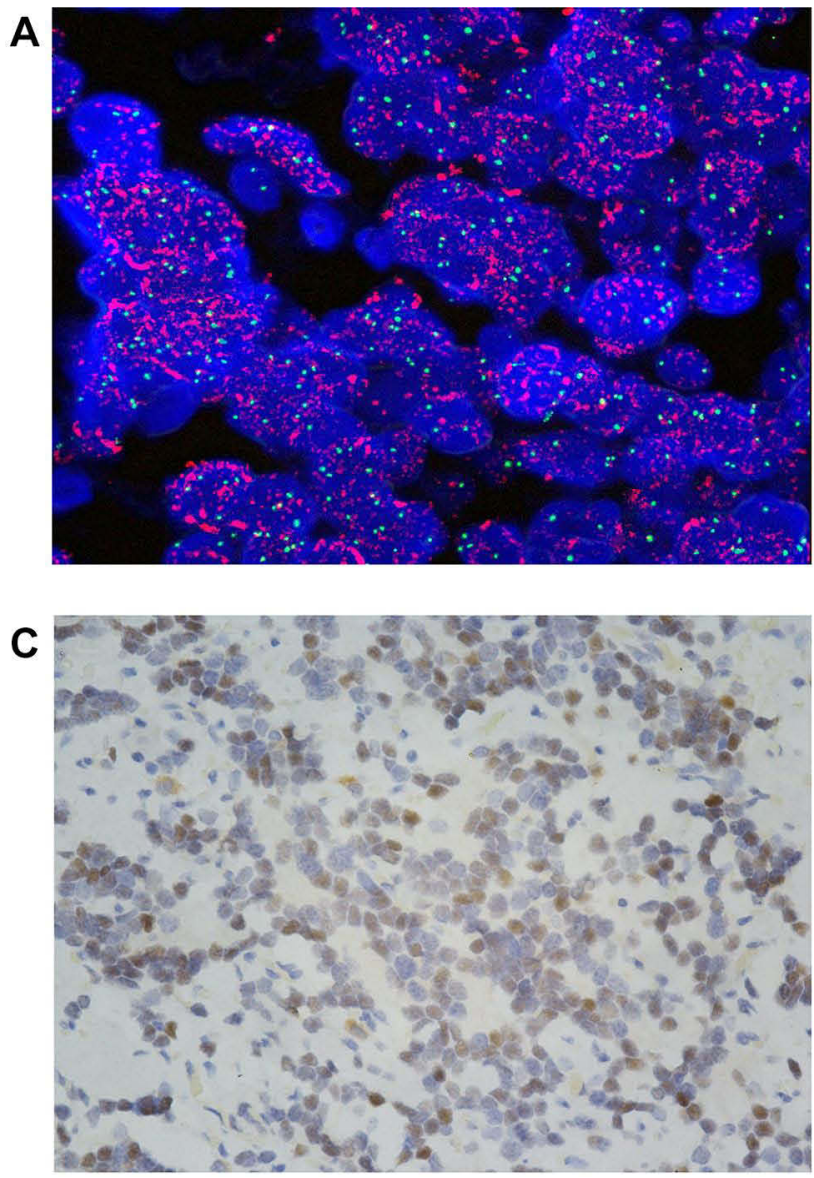

B

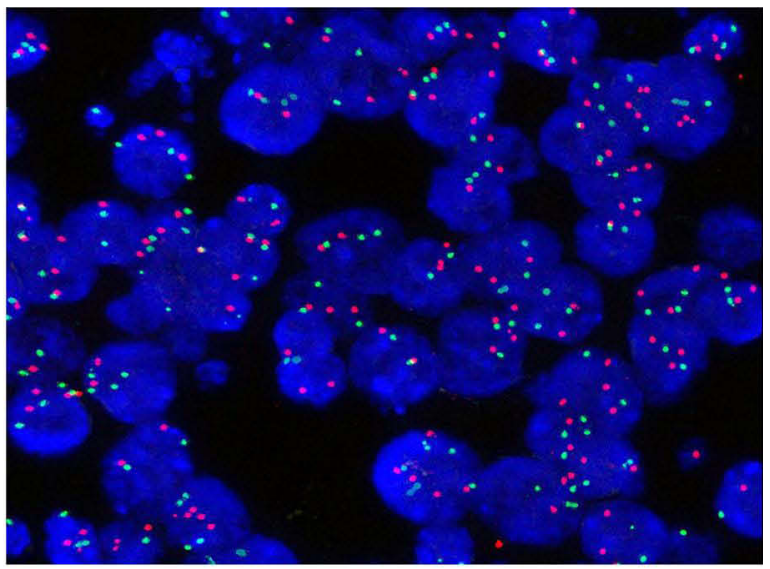

D

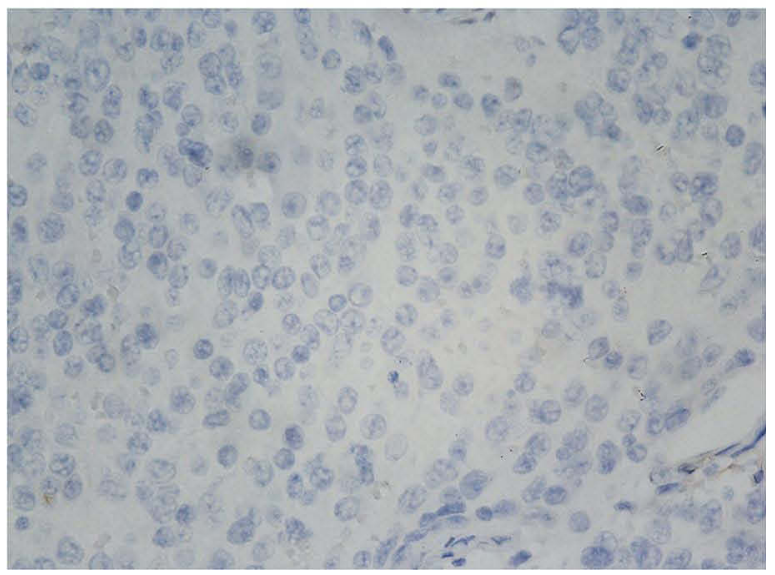

Figure I MYCN gene expression detected by FISH and c-MYC protein expression by IHC in NTs. (A) MYCN amplification, FISH, original magnification $\times$ I000. MYCN (red signal) clustering occurred. (B) Lack of MYCN amplification, FISH, original magnification $\times 1000$. The ratio of MYCN (red signal) to LAF (green signal) was $\leq 1.0$. (C) Positive (+) c-MYC expression, immunostaining, original magnification $\times 400$. (D) Negative (-) c-MYC expression, immunostaining, original magnification $\times 400$. 
Table 2 Association of MYCN Amplification and c-MYC Expression with the Shimada Classification

\begin{tabular}{|l|l|l|l|l|}
\hline & $\begin{array}{l}\text { MYCN } \\
\text { (Amplified) }\end{array}$ & $\begin{array}{l}\text { MYCN } \\
\text { (Not Amplified) }\end{array}$ & $\begin{array}{l}\text { c-MYC } \\
(+)\end{array}$ & $\begin{array}{l}\text { c-MYC } \\
(-)\end{array}$ \\
\hline $\begin{array}{l}\text { FH type } \\
\text { uFH type }\end{array}$ & 2 & 119 & 13 & 108 \\
$\begin{array}{l}P \text { value } \\
<1.5 \text { year-old }\end{array}$ & 0.0001 & 30 & 3 & 53 \\
$\geq 1.5$ year-old & 27 & 53 & 0.3977 & \\
$P$ value $^{a}$ & 0.0001 & 96 & 4 & 50 \\
\hline
\end{tabular}

Notes: a From Fisher's test.

0.104, SE of Kappa $=0.030,95 \%$ confidence interval $=-$ $0.163 \sim 0.045$, weighted Kappa $=-0.110$ ], suggesting that the results of the two methods did not overlap.

\section{Association of MYCN and c-MYC with the Shimada Classification}

As shown in Table 2, for the cases with amplified MYCN, two cases had FH, while the others had uFH. After analysis
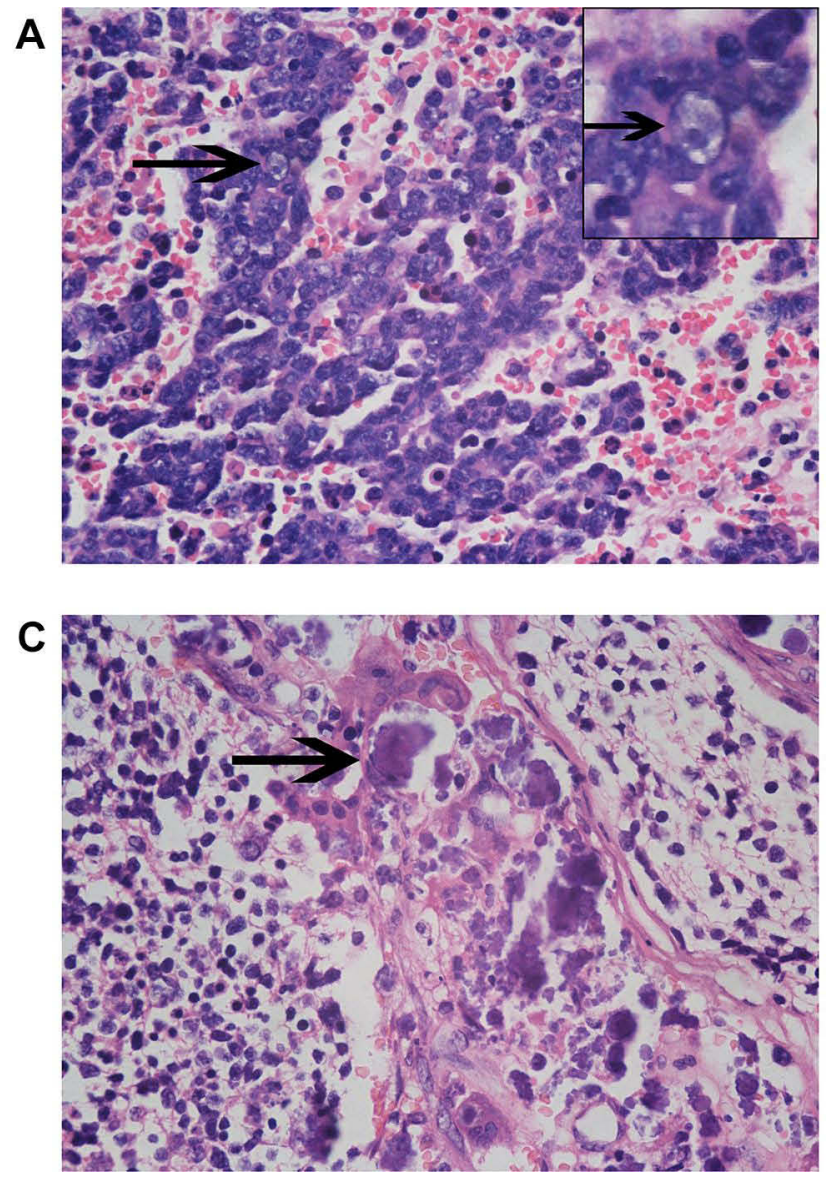

by Fisher's test, a statistically significant difference was observed between the FH and uFH types in the cases with $M Y C N$ amplification $(P=0.0001)$, indicating that amplified $M Y C N$ mainly occurred in the uFH type. Moreover, only one patient was $<1.5$ years old, and a statistically significant difference was found in age via Fisher's test $(P=0.0001)$, indicating that amplified $M Y C N$ was mainly observed in such patients. The mitosis-karyorrhexis index (MKI) of all cases was above $2 \%$, classified as high or intermediate.

For the cases with positive c-MYC, three had $\mathrm{uFH}$ and the others FH. Fisher's test suggested that no statistically significant difference was found between the types in these cases $(P=0.3977)$. Four of the positive cases were less than one year and six months old, while the remaining cases were all older than one year and six months old. Less than 1 year and 6 months, other ages are more than 1 year and 6 months. According to Fisher's test, $\mathrm{P}$ is equal to 0.7790 . There was no significant difference in age distribution. MKI was less than $4 \%$, indicating medium or low MKI.
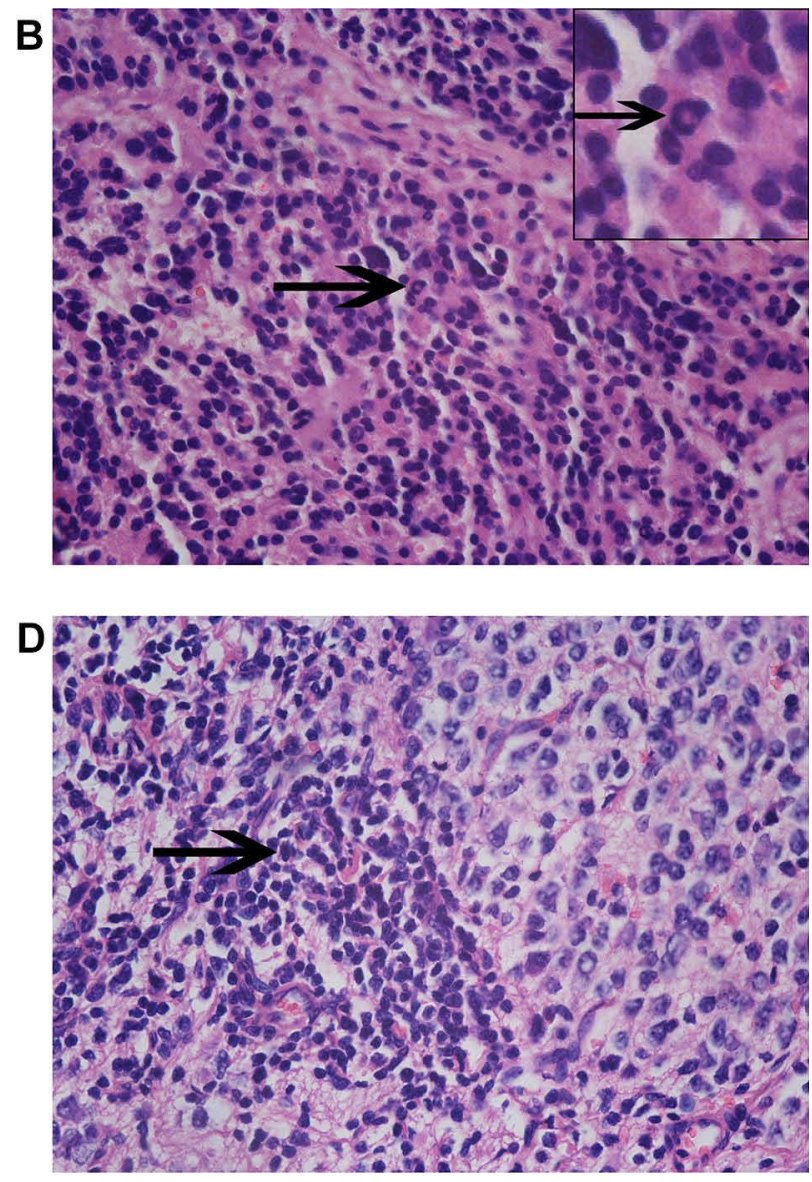

Figure 2 Morphological changes of NTs. HE staining, original magnification $\times 400$. (A) Bull's eye cells (indicated by arrows). (B) Nuclear inclusions (indicated by arrows). (C) Calcification (indicated by arrows). (D) Lymphocyte clustering (indicated by arrows). 


\section{Association of MYCN and c-MYC with Morphological Characteristics}

Morphological changes, such as "bull's eye" cells and nuclear inclusions, were observed in the cases with poor prognosis with $M Y C N$ amplification but not in those with positive c-MYC expression. Changes that suggest a good prognosis, such as calcification and lymphocyte clustering, were only found in the one case with positive c-MYC expression (Figure 2, Table 3).

\section{Association of MYCN and c-MYC with Prognosis}

Table 4 shows the three-year survival rate in the patients with NTs. The $\chi^{2}$ test showed that patients with the FH type with amplified $M Y C N$ or positive c-MYC had a lower survival rate than those without amplified $M Y C N$ or with negative c-MYC $(P=0.0001)$.

Survival analyses were performed using Kaplan-Meier curves and Log rank tests (Figure 3). The results showed that there was a significant difference in survival rate between the c-myc positive and negative groups $(\mathrm{P}=0.001)$. Cox Proportional Hazards Model Survival Analysis showed that the model test was meaningful (Score $=11.446, \mathrm{P}=0.001 ; \chi 2=8.121, \mathrm{P}=0.004$ ), there was significant difference in survival rate between the c-myc positive group and the negative group $(\mathrm{P}=0.001)$. Multivariate Cox Proportional Hazards Model Survival Analysis showed that Model test was significant (Score $=67.756, \mathrm{P}=0.000 ; \chi 2=55.368, \mathrm{P}=0.000$ ), c-myc was an independent influencing factor $(\mathrm{P}=0.000)$.

\section{Discussion}

In clinical practice, some patients with early stage and low-risk NTs experience a poor chemotherapeutic effect, ie, tumors are prone to metastasize and relapse during treatment. The reason might be resistance to chemotherapeutic agents ${ }^{11,12}$ or detection inaccuracy of the samples. However, even though there are many oncobiology-related factors, current understanding of NTs is an ongoing process. ${ }^{13,14}$
Table 4 Association of MYCN Amplification and c-MYC Expression with the 3-Year Survival Rate in Patients with NTs

\begin{tabular}{|l|l|l|l|l|}
\hline & MNA & Non-MNA & c-MYC (+) & c-MYC (-) \\
\hline FH type & $46 \%$ & $90 \%$ & $43 \%$ & $87 \%$ \\
uFH type & $41 \%$ & $53 \%$ & $44 \%$ & $55 \%$ \\
\hline
\end{tabular}

Abbreviations: MNA, MYCN amplification; non-MNA, cases without MYCN amplification.

Currently, morphological subtypes, molecular tests, and clinical stages of NTs are comprehensively considered to judge the prognosis. The most widely adopted molecular test is the FISH analysis of MYCN amplification. Years of clinical observation have confirmed that cases with amplified MYCN have specific morphological characteristics and a poorer prognosis compared with those without amplified $M Y C N{ }^{15}$ However, in our study, several cases without $M Y C N$ amplification still had a poor prognosis. Moreover, several subtypes of morphological changes associated with oncobiology have been found to date, but morphological changes in patients with $\mathrm{FH}$ and $\mathrm{uFH}$ types according to the Shimada classification are more commonly recognized, which makes a preliminary evaluation of the prognosis in patients with NTs.

The appearance of "bull's eye" cells, ie, tumor cells with large, round, and red nucleoli, is a morphological change indicating strong tumor infiltration. ${ }^{15,16}$ The nuclear chromatin of the cells is presented as small pieces partially adhering to the inside of the nuclear membrane. This study revealed that such cells were found in most of the cases with MYCN gene amplification. ${ }^{16}$ Nuclear inclusions, ie, uncommon structures in NT cells, are formed after the cytoplasm is filled in the nuclei and mainly observed in cell samples of undifferentiated and poorly differentiated NTs. Calcification and lymphocyte clustering are common morphological changes in patients with a good prognosis, ${ }^{17}$ often appearing in cases with differentiated NTs and a low MKI. However, determining the prognosis in patients with NTs is limited by the morphological characteristics of the tumor since patients with FH type with a poor prognosis may also have

Table 3 Association of MYCN Amplification and Positive c-MYC Expression with Morphological Changes

\begin{tabular}{|l|l|l|l|l|}
\hline \multirow{2}{*}{} & \multicolumn{2}{|l|}{ Morphological Changes Related to Poor Prognosis } & \multicolumn{2}{l|}{ Morphological Changes Related to Good Prognosis } \\
\cline { 2 - 5 } & Bull's Eye Cells & Nuclear Inclusions & Calcification & Lymphocyte Clustering \\
\hline MYCN (Amplified) & $26 / 28$ & $3 / 28$ & 0 & 0 \\
C-MYC (+) & 0 & 0 & 0 & $1 / 16$ \\
\hline
\end{tabular}




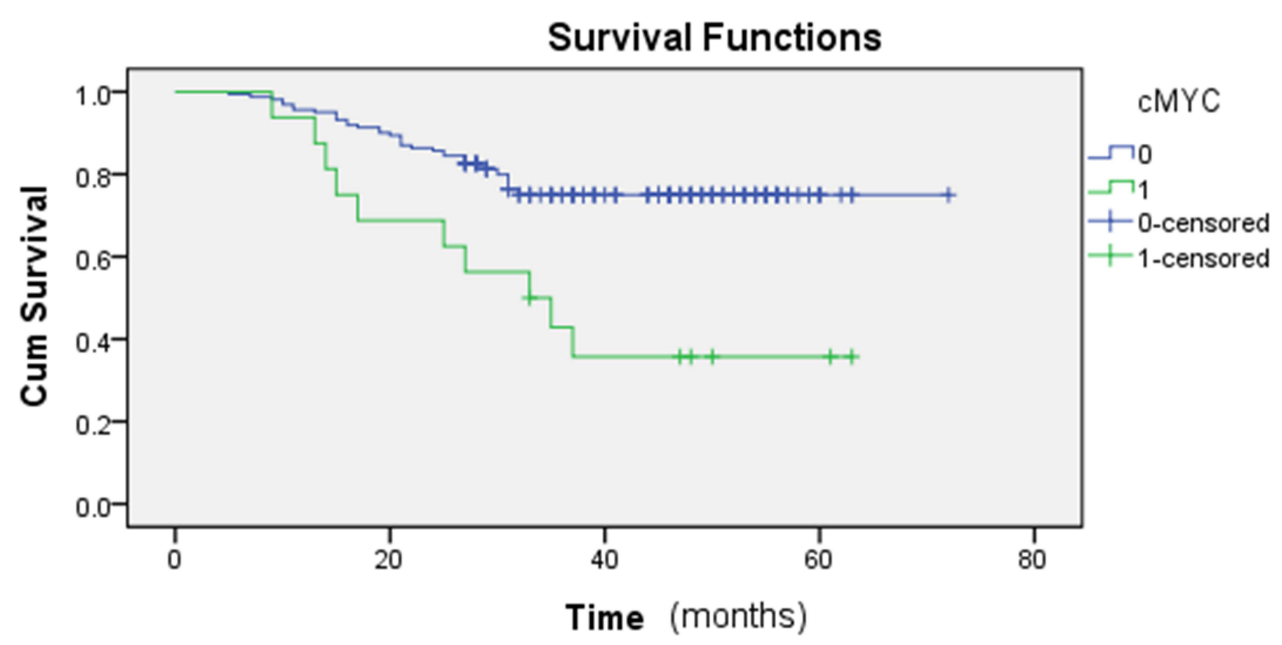

Figure 3 Kaplan-Meier curves and Log rank tests. The results showed that there was a significant difference in survival rate between the c-myc positive and negative groups $(P=0.001)$.

calcification and lymphocyte clustering in NTs. Therefore, other methods for prognosis are required to supplement.

In this study, of all the patients positive for c-MYC, three had uFH and the others FH. No significant difference was found in age, and the MKI for all cases (less than 4\%) was classified as intermediate or low. "Bull's eye" cells, nuclear inclusions, and calcification were not found in any of the cases, and obvious lymphocyte clustering was observed in only one case. Amplified MYCN was not present in all cases. The 3-year survival rate and survival time of patients with positive c-myc were significantly lower than those with negative c-myc. This indicates that although the patients with positive c-myc have poor prognosis, cell morphology does not show the characteristics of strong tumor infiltration, and tissue types are more common in FH type.

In conclusion, compared with cases with amplified $M Y C N$, which are usually the uFH type or commonly found to be morphologically heterogeneous, there was no significant difference between the $\mathrm{FH}$ and $\mathrm{uFH}$ types in the positive c-MYC cases. Furthermore, cases with positive c-MYC had a much lower three-year survival rate than those with negative c-MYC. Thus, it is evident that c-MYC is often expressed in patients with a good prognosis as determined by morphological classification and MYCN detection. Kaplan-Meier curves and other statistical methods showed that the survival time of patients with positive c-myc was significantly less than that of patients with negative c-myc. Cox Proportional Hazards Model Survival Analysis suggested that c-myc expression was not affected by Shimada typing, age and MyCN amplification results. Accordingly, c-MYC protein expression could be a significant independent indicator of prognosis in patients with NTs and may be used as an important supplementary prognostic method aside from $M Y C N$ gene amplification detection.

\section{Ethics Approval and Consent to Participate}

This study was carried out within an appropriate ethical framework. The patients' parents understood the scientific research uses, and all experimental procedures were approved by the Biomedical Ethics Committee of Chongqing Medical University. This study was conducted in accordance with the declaration of Helsinki. Written informed consent was obtained from all participants.

\section{Consent for Publication}

All patient guardians signed a document of informed consent.

\section{Acknowledgments}

We would like to acknowledge the hard and dedicated work of all the staff that implemented the intervention and evaluation components of the study.

\section{Funding}

Joint project of Chongqing Science and Technology Commission and Health Commission,2020MSXM040.

\section{Disclosure}

The authors declare that they have no competing interests. 


\section{References}

1. Shimada $\mathrm{H}$, Umehara S, Monobe $\mathrm{Y}$, et al. International neuroblastoma pathology classification for prognostic evaluation of patients with peripheral neuroblastic tumors: a report from the Children's Cancer Group. Cancer. 2001;92(9):2451-2461. doi:10.1002/1097-0142(20011101)92:9<2451::AID-CNCR1595>3.0.CO;2-S

2. Wang H, Mejia MC, Gonzalez SJ, Zoorob RJ, Chai W, Du XL. Cancer incidence and survival trends among infants in the United States from 1975 to 2014. Pediatr Blood Cancer. 2021;68(4):e28917. doi:10.1002/ pbc. 28917

3. Shimada H, Ikegaki N. Neuroblastoma pathology and classification for precision prognosis and therapy stratification. Neuroblastoma. 2019. doi:10.1016/B978-0-12-812005-7.00001-1

4. Shimada H, Ambros IM, Dehner LP, Hata J, Joshi VV, Roald B. Terminology and morphologic criteria of neuroblastic tumors: recommendations by the International Neuroblastoma Pathology Committee. Cancer. 1999;86:349-363. doi:10.1002/(SICI)1097-0142(19990715) $86: 2<349::$ AID-CNCR20 $>3.0 . \mathrm{CO} ; 2-\mathrm{Y}$

5. Cheung NK, Dyer MA. Neuroblastoma: developmental biology, cancer genomics and immunotherapy. Nat Rev Cancer. 2013;13:397-411. doi:10.1038/nrc3526

6. Berthold F, Spix C, Kaatsch P, Lampert F. Incidence, survival, and treatment of localized and metastatic neuroblastoma in Germany 1979-2015. Paediatr Drugs. 2017;19:577-593. doi:10.1007/s40272-017-0251-3

7. Huang M, Weiss WA. Neuroblastoma and MYCN. Cold Spring Harb Perspect Med. 2013;3:a014415. doi:10.1101/cshperspect.a014415

8. Teshiba R, Kawano S, Wang LL, et al. Age-dependent prognostic effect by mitosis-karyorrhexis index in neuroblastoma: a report from the Children's Oncology Group. Pediatr Dev Pathol. 2014;17:441-449. doi:10.2350/14-06-1505-OA.1

9. Wang LL, Teshiba R, Ikegaki N, et al. Augmented expression of MYC and/or MYCN protein defines highly aggressive MYC-driven neuroblastoma: a Children's Oncology Group study. $\mathrm{Br} J$ Cancer. 2015;113:57-63. doi:10.1038/bjc.2015.188
10. Wang LL, Suganuma R, Ikegaki N, et al. Neuroblastoma of undifferentiated subtype, prognostic significance of prominent nucleolar formation, and MYC/MYCN protein expression: a report from the Children's Oncology Group. Cancer. 2013;119:3718-3726. doi:10. $1002 /$ cncr.28251

11. Moreno L, Rubie H, Varo A, et al. Outcome of children with relapsed or refractory neuroblastoma: a meta-analysis of ITCC/SIOPEN European Phase II clinical trials. Pediatr Blood Cancer. 2017;64 (1):25-31. doi:10.1002/pbc.26192

12. Pajic M, Norris MD, Cohn SL, Haber M. The role of the multidrug resistance-associated protein 1 gene in neuroblastoma biology and clinical outcome. Cancer Lett. 2005;228(1-2):241-246. doi:10.1016/ j.canlet.2005.01.060

13. Tomolonis JA, Agarwal S, Shohet JM. Neuroblastoma pathogenesis: deregulation of embryonic neural crest development. Cell Tissue Res. 2018;372(2):245-262. doi:10.1007/s00441-017-2747-0

14. Salazar BM, Balczewski EA, Ung CY, Zhu S. Neuroblastoma, a paradigm for big data science in pediatric oncology. Int $J$ Mol Sci. 2016;18(1):37. doi:10.3390/ijms 18010037

15. Suganuma R, Wang LL, Sano H, et al. Peripheral neuroblastic tumors with genotype-phenotype discordance: a report from the Children's Oncology Group and the International Neuroblastoma Pathology Committee. Pediatr Blood Cancer. 2013;60(3):363-370. doi:10.10 02/pbc. 24238

16. Ambros IM, Hata J, Joshi VV, et al. Morphologic features of neuroblastoma (Schwannian stroma-poor tumors) in clinically favorable and unfavorable groups. Cancer. 2002;94(5):1574-1583. doi:10.10 02/cncr. 10359

17. Mina M, Boldrini R, Citti A, et al. Tumor-infiltrating T lymphocytes improve clinical outcome of therapy-resistant neuroblastoma. Oncoimmunology. 2015;4(9):e1019981. doi:10.1080/2162402X.20 15.1019981
International Journal of General Medicine

\section{Publish your work in this journal}

The International Journal of General Medicine is an international, peer-reviewed open-access journal that focuses on general and internal medicine, pathogenesis, epidemiology, diagnosis, monitoring and treatment protocols. The journal is characterized by the rapid reporting of reviews, original research and clinical studies

\section{Dovepress}

across all disease areas. The manuscript management system is completely online and includes a very quick and fair peer-review system, which is all easy to use. Visit http://www.dovepress.com/ testimonials.php to read real quotes from published authors. 